\title{
L-Histidinium Maleate Crystals for Nonlinear Frequency Conversion
}

\author{
C. Alosious Gonsago, S. Pandi, Helen Merina Albert, and A. Joseph Arul Pragasam
}

\begin{abstract}
A new amino acid organic nonlinear optical material L-histidinium maleate (LHM), has been synthesized and successfully grown from aqueous solution by slow solvent evaporation method at room temperature. The solubility of the compound was measured in aqueous solution at different temperatures. The grown crystal was subjected to single crystal XRD and powder XRD studies in order to identify the structural arrangement. The chemical composition of the grown crystal was confirmed by Energy dispersive $X$-ray analysis (EDAX). The percentage of optical transmittance by the grown crystal was identified by UV-visible spectral study. The nonlinear optical property of the grown material was identified by the powder technique of Kurtz and Perry. The HR-SEM analysis shows the surface morphology of the grown crystal. The thermal stability of the grown crystal was found by differential thermal analysis and differential scanning calorimetry.
\end{abstract}

\section{Index Terms-DTA, DSC, EDAX, HR-SEM, NLO, XRD.}

\section{INTRODUCTION}

Nonlinear optics is a new frontier in the field of science and technology which plays an important role in the emerging era of photonics. Nonlinear optical (NLO) materials have been extensively studied in the recent years, due to their potential applications in various fields like optical data storage, optical switching, image processing and manipulation [1]-[3]. Materials with large second-order optical nonlinearities, good optical transparency, short lower cut-off wavelengths, and stable physicochemical performances are required in order to realize many of these applications [4]. Organic nonlinear materials are drawing a great deal of attention, due to their potential nonlinearities and rapid response in electro-optic effect when compared with the inorganic nonlinear materials. Recently, a number of organic nonlinear optical materials have been reported in literature owing to their nonlinear optical and photonic applications [5]-[7]. Among these classes of materials, amino acids are interesting and useful materials for NLO applications.

The salts of basic amino acid L-histidine gain much interest as promising nonlinear optical materials after the

Manuscript received December 22, 2011; revised January 26, 2012.

C. Alosious Gonsago is with the Physics Department, Faculty of Science and Humanities, A.J. College of Engineering, Affiliated to Anna University, Chennai, India, (email: c.alosious@gmail.com).

S. Pandi was with the, PG \& Research Department of Physcs, Presidency College, Chennai, India, (email: pandisangu@gmail.com).

H. M. Albert and A. J. A. Pragasam are with the, Physics Department, Sathyabama University, Chennai-600119, India, (email: merinagonsago@gmail.com,drjosephsu@gmail.com). early works of Marcy et al., that the nonlinearity of L-histidine tetraflouroborate is much greater than that of potassium dihydrogen phosphate [8]. Due to its basic nature L-histidine forms a number of salts with different organic and inorganic acids which have shown NLO properties. Maleic acid, basically a dicarboxylic acid with large $\pi$-conjugation has attracted a great deal of attention [9]. Based on the previous reports of L-histidine salts and their NLO properties, we have successfully synthesized L-histidinium maleate, a new analog of L-histidine salt. It is observed that several authors have followed the spectral, optical, and thermal studies for material characterization [10]-[15]. In this paper, we report the crystal growth and its characterization by XRD, energy dispersive X-ray analysis, UV-Visible, NLO, HR-SEM, differential thermal analysis (DTA), and differential scanning calorimetry (DSC).

\section{MATERIAL AND METHODS}

\section{A. Solubility Study}

AR grade of L-histidine and maleic acid were used for the solubility measurements and crystal growth. Aqueous solution of LHM was prepared by dissolving equimolar ratio of L-histidine and maleic acid in double distilled water. Here, the solubility is the amount of solute (gram) present in $100 \mathrm{ml}$ of saturated solution at certain temperature. The solubility study is generally carried out to find out the quantity of the material available for the crystal growth. The solubility curve of LHM sample in double distilled water at different temperatures ranging from 30 to $50{ }^{\circ} \mathrm{C}$ is shown in Fig. 1 . From the curve, it is noted that solubility increases with increase in temperature. The sample of this study has positive temperature coefficient of solubility and hence, the LHM crystals can be grown from aqueous solution by slow solvent evaporation method.

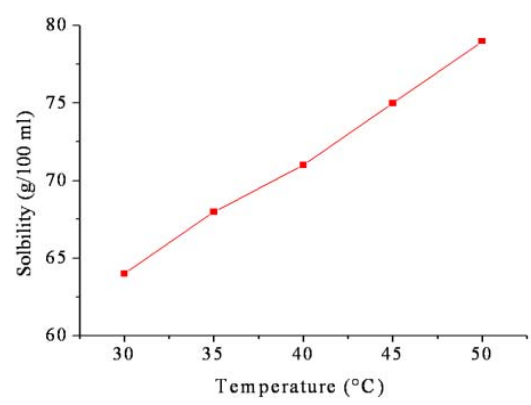

Fig. 1. Solubility curve of LHM sample.

\section{B. Crystal Growth}

According to the solubility data, the title compound L-histidinium maleate (LHM) has been grown by dissolving 
1:1 molar ratio of L-histidine and maleic acid in double distilled water at room temperature $\left(32^{\circ} \mathrm{C}\right)$. The salts were stirred well for nearly $2 \mathrm{~h}$ using a magnetic stirrer in order to obtain a uniform mixture of the solution over the entire volume. The substance was purified by successive crystallization process. After recrystallization process, saturated solution was prepared at $32{ }^{\circ} \mathrm{C}$ using the synthesized salts. The solution was finally filtered twice or thrice using micro-whatmann filter papers to eliminate unwanted impurities. Two drops of $\mathrm{H}_{2} \mathrm{O}_{2}$ were added to the mother solution to inhibit the growth of any microorganism [16]. The filtered solution was kept in a crystallizing vessel, covered with a perforated sheet and placed in a dust free atmosphere for constant growth. A good quality single crystal of optimum size was obtained in a period of 25 days at room temperature. The photograph of as grown crystal of LHM crystal is shown in Fig. 2.

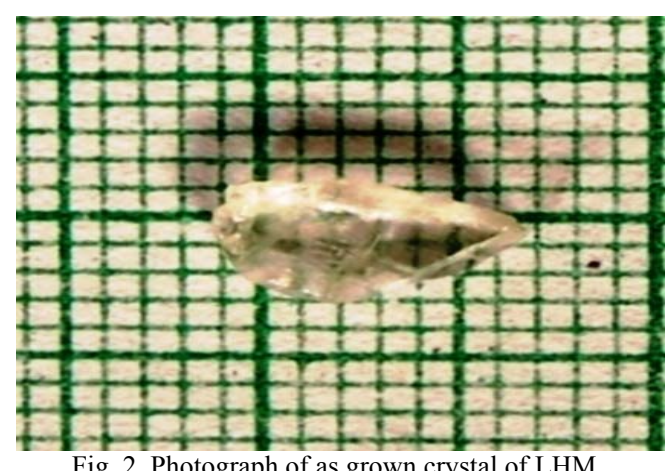

\section{RESULT AND DISCUSSION}

\section{A. XRD Studies}

Single crystal XRD and powder XRD studies were carried out for the grown crystal, in order to find the crystal structure and lattice parameters. The single crystal X-ray diffraction analysis was carried out using BRUKER AXS kappa apex2 CCD diffractometer. The unit cell dimensions were measured using MoK $\alpha$ radiation source of wavelength $0.7107 \AA$. The $X$-ray crystallography data shows that the crystal belongs to monoclinic system with $\mathrm{P} 21$ space group, $Z=4$. The molecular formula of the compound is $\mathrm{C} 10 \mathrm{H} 16 \mathrm{~N} 3 \mathrm{O} 7.5$. The observed values of lattice parameters are $\mathrm{a}=11.4656(7) \AA \mathrm{,}, \mathrm{b}$ $=8.0530(5) \AA, c=14.9705(9) \AA, \quad V=1353.75(14) \AA 3, \alpha=\gamma$ $=90^{\circ}$ and $\beta=101.657^{\circ}$.

The grown crystal was characterized by X-ray powder diffraction technique using a JEOL JDX services instrument with $\mathrm{CuK} \alpha$ radiation source of wavelength $\lambda=1.5406 \AA$. The grown crystal was crushed to a uniform fine powder and subjected to powder X-ray diffraction. The $2 \theta$ range analyzed was from $10^{\circ}$ to $70^{\circ}$ by employing continuous scan mode. The powder XRD pattern of the sample is depicted in the Fig. 3. The sharp and well defined peaks at specific 2-theta values indicate good crystalline nature and purity of the sample.

\section{B. EDAX Analysis}

Energy dispersive $X$-ray analysis (EDAX) is a micro-analytical technique which is used to obtain useful information regarding the chemical composition of the grown crystal. In this work, the grown crystal was subjected to
EDAX analysis using the instrument FEI QUANTA 200F energy dispersive X-ray micro analyzer. A fine beam of $\mathrm{X}$-rays was made to fall into the LHM sample. The number and energy of the X-rays emitted by the sample was measured by an energy-dispersive spectrometer. Since the energy of the X-rays emitted from the sample is attributed to the energy difference between the two shells and of the atomic structure of the compound, the elemental composition of the specimen can be measured. The EDAX spectrum of the crystal is depicted in Fig. 4. The weight percentages (wt \%) of $\mathrm{C}, \mathrm{N}$ and $\mathrm{O}$ as obtained from EDAX analysis are in concurrent with the theoretical values and are listed in TABLE I.

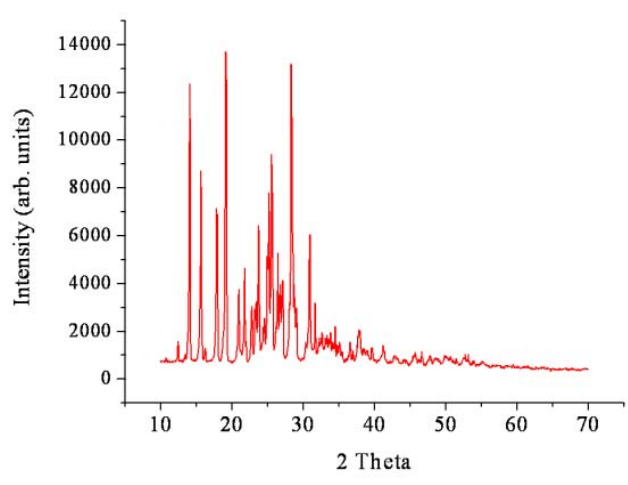

Fig. 3. Powder XRD pattern of LHM crystal.

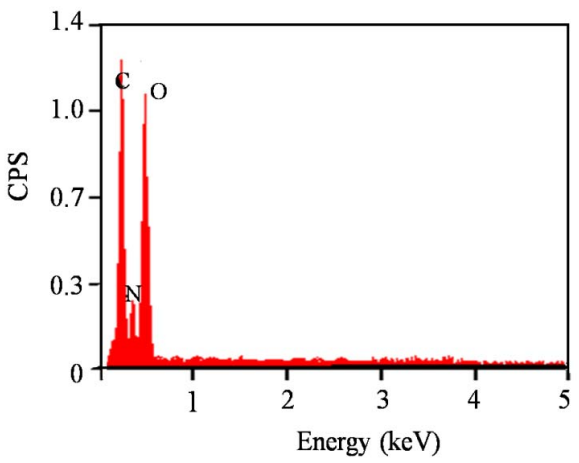

Fig. 4. EDAX spectrum of LHM crystal.

TABLE I: EDAX QUANTIFICATION TABLE OF LHM

\begin{tabular}{ccc}
\hline \hline Element & $\begin{array}{c}\text { Wt (\%) } \\
\text { Experimental }\end{array}$ & $\begin{array}{c}\text { Wt (\%) } \\
\text { Theoretical }\end{array}$ \\
\hline Carbon & 43.32 & 42.58 \\
Nitrogen & 14.85 & 14.89 \\
Oxygen & 41.83 & 42.53 \\
\hline \hline
\end{tabular}

\section{Optical Analysis}

The optical transmittance study is an effective tool in identifying the usefulness of a nonlinear optical material in the visible and blue regions. The UV-visible transmittance spectrum was recorded for the grown crystal in the wavelength range of 200-1000 nm using a Varian Carry-5E UV-vis Spectrophotometer. The optical transmittance spectrum of LHM is depicted in Fig. 5. From the transmittance spectrum, it is observed that the grown crystal is completely transparent in the UV and visible spectral regions with the lower cut off wavelength at around $280 \mathrm{~nm}$, 
thereby confirming the advantages of the crystal for photonics applications. Complete transparency of the crystal between the region $280-1000 \mathrm{~nm}$ is an important prerequisite for NLO applications [17]. It is well known that optical absorption in the near UV region arises from the electronic transitions associated within the crystal. The optical band gap of the crystal is calculated using the formula $\mathrm{Eg}=1240 / \lambda(\mathrm{nm})$ and is found to be $4.43 \mathrm{eV}$. Hence, due to wide band gap, the grown crystal has wide transmittance window in the visible and UV regions.

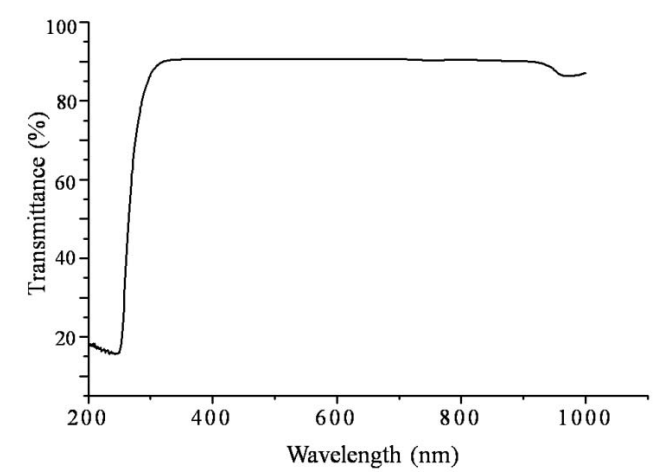

Fig. 5. UV-Visible transmittance spectrum of LHM crystal.

\section{NLO Property}

The nonlinear property of LHM crystal was studied by employing Kurtz and Perry powder technique [18]. In this technique, the grown sample was powdered into fine microcrystalline samples and then densely packed between two transparent glass slides. A Q-switched mode Nd:YAG laser operated at the fundamental wavelength $1064 \mathrm{~nm}$ with 8 ns pulse width and $10 \mathrm{~Hz}$ pulse rate was used as the radiation source. The experimental set-up of second harmonic generation was reported elsewhere [19]. The laser beam was allowed to pass through the sample cell. The output beam from the sample was filtered by an IR detector and then identified by a photomultiplier tube. A color filter was used to absorb IR radiation and transmit that of second harmonic radiation of wavelength $532 \mathrm{~nm}$. The final output was displayed on the oscilloscope. The frequency conversion efficiency of the crystal was confirmed by the emission of green radiation from the sample.

\section{E. HR-SEM Analysis}

In order to analyze the nature and surface morphology of the grown crystal, HR-SEM analysis was employed. The crystal was cut into few $\mathrm{mm}$ for observing the surface morphology. The highly transparent region of the crystal was chosen for analysis. The SEM images of LHM crystal taken in different magnifications are shown in Fig. 6. The images show step-like growth, which suggests the existence of grain boundaries and striations. The micrographs also show the presence of few cracks and visible inclusions on the surface of the crystal. This may be due the temperature oscillations during the crystal growth.

\section{F. DTA Analysis}

Differential Thermal Analysis (DTA) is a very popular thermal analysis technique used to find endothermic and exothermic transitions as a function of temperature. The DTA provides useful information regarding the transformations that have occurred, water of crystallization and melting point of the compound [20]. The DTA analysis was employed using the instrument NETSZCH STA $409 \mathrm{C} / \mathrm{CD}$ under nitrogen atmosphere between the temperature range 20-1200 ${ }^{\circ} \mathrm{C}$ in steps of $10{ }^{\circ} \mathrm{C}$. The typical DTA curve of LHM crystal is shown in Fig. 7. The DTA curve shows a sharp endothermic peak at around $120{ }^{\circ} \mathrm{C}$ which is attributed to water of crystallization. This is followed by two consecutive peaks at around $139{ }^{\circ} \mathrm{C}$ and $279{ }^{\circ} \mathrm{C}$ which are attributed to the decomposition and volatilization of the compound. Although the melting point of the compound is around $139{ }^{\circ} \mathrm{C}$, the compound loses its structure at around $120^{\circ} \mathrm{C}$ due to the presence of water molecules.
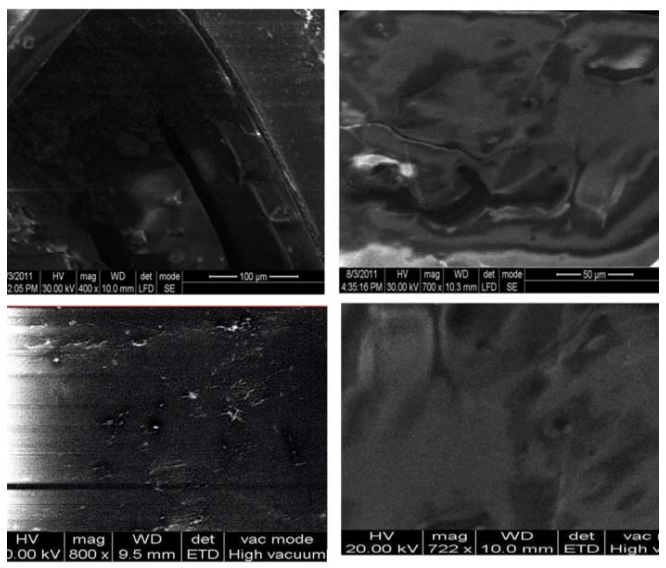

Fig. 6. SEM images of LHM crystal.

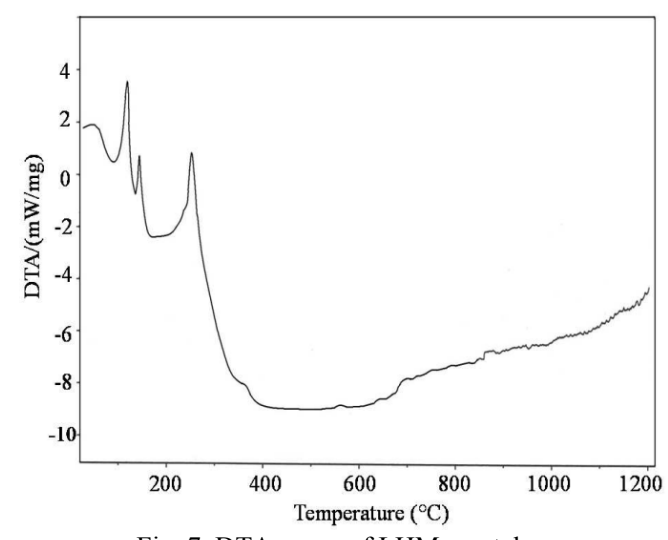

Fig. 7. DTA curve of LHM crystal.

\section{G. DSC Analysis}

The differential scanning calorimetry (DSC) is a thermo-analytical technique used to determine melting temperature, water of crystallization and heat of fusion. Differential scanning calorimetry measures heat flow to or from the material as a function of temperature and time. The DSC analysis was carried out for the LHM crystal between $25{ }^{\circ} \mathrm{C}$ and $200{ }^{\circ} \mathrm{C}$ at a heating rate of $10{ }^{\circ} \mathrm{C} / \mathrm{min}$ in the nitrogen atmosphere using the instrument NETZSCH DSC 204. The typical DSC curve of LHM is shown in Fig. 8. The DSC curve shows almost the same changes shown by DTA curve. The curve is smooth up to $110^{\circ} \mathrm{C}$ and then shows two sharp endothermic peaks at $117.5^{\circ} \mathrm{C}$ and $136{ }^{\circ} \mathrm{C}$ respectively. The first peak is due to the removal of weakly entrapped lattice water from the crystal and the second peak is due to the 
partial decomposition of the compound. The DSC analysis clearly indicates that the grown crystal is hydrated in nature and is found to be thermally stable up to $117.5^{\circ} \mathrm{C}$. The smoothness of the DSC curves reveals the purity of the crystal.

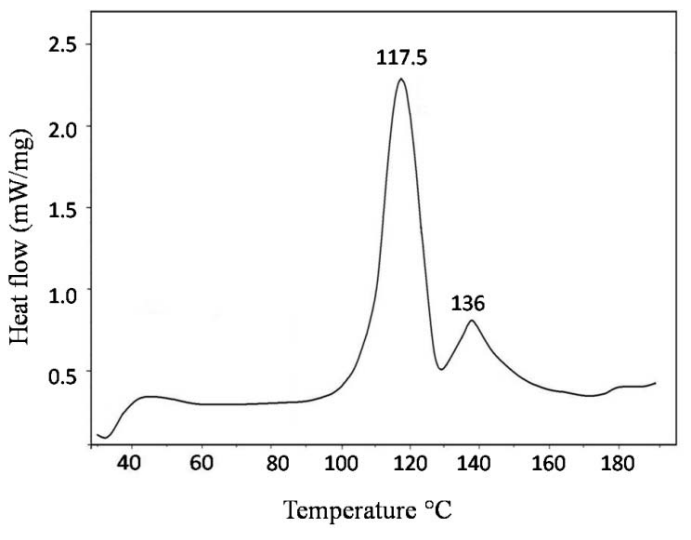

Fig. 8. DSC curve of LHM crystal.

\section{CONCLUSION}

Good quality single crystal of L-Histidinium maleate has been grown successfully by slow evaporation solution growth technique at room temperature. Single crystal and powder X-ray crystallographic data indicate that the LHM crystal belongs to monoclinic system with the space group $\mathrm{P} 21$. The chemical composition of the grown crystal was ascertained by EDAX analysis. The UV-visible transmittance spectrum reveals the transparency of the crystal which shows that the percentage of optical transmittance is much higher in the range 280 to $1000 \mathrm{~nm}$. The emission of SHG from the grown crystal was confirmed by the emission of green radiation using Kurtz and Perry powder method. The HR-SEM analysis reveals the existence of grain boundary and striations on the surface of the grown crystal. The thermal stability of LHM crystal was analyzed by DTA and DSC techniques which indicate that the crystal is stable upto $117.5^{\circ} \mathrm{C}$ and it can be used as potential material for nonlinear optical applications below this temperature.

\section{ACKNOWLEDGMENT}

The authors sincerely thank Sophisticated Analytical Instrument Facility (SAIF), IIT-Madras and Sathyabama University, Chennai for providing experimental facilities. The authors also acknowledge the Department of Physics, B.S. Abdur Rahman University, Chennai for extending the NLO characterization facility.

\section{REFERENCES}

[1] S. Moitra, S. K. Seth, and T. Kar, "Synthesis, crystal structure characterization and DFT studies of L-valine L-valinium hydrochloride," Journal of Crystal Growth 312, 1977-1982 (2010).
[2] C. A. Gonsago, H. M. Albert, P. Malliga, and A. J. A. Pragaam, "Crystallization, spectral, and thermal characterization of L-histidine mehyl ester dihydrochloride (LHMED)," Journal of Thermal Analysis and Calorimetry DOI 10.1007/s10973-011-1719-y (2011) Article in press.

[3] G. A. Babu and P. Ramasamy, "Growth and characterization of an organic NLO material Ammonium malate," Current Applied Physics, vol. 10, pp. 214-220, 2010.

[4] M. Vimalan, T. R. Kumar, S. Tamilselvan, P. Sagayaraj, and C. K. Mahadevan, "Growth and properties of novel organic nonlinear optical crystal: 1-alaninium tartrate (LAT)," Physica B 405, pp. 3907-3913, 2010.

[5] M. Rajasekaran, P. Anbusrinivasan, and S. C. Mojumdar,"Growth, spectral and thermal characterization of 8-hydroxyquinoline," Journal of Thermal Analyis and Calorimetry, vol. 100, pp. 827-830, 2010.

[6] S. Moitra and T. Kar, "Growth and characterization of L-valine- a nonlinear optical crystal," Crystal Research Technology 45, pp. 70-74, 2010.

[7] A. S. Gill and S. Kalainathan, "Thermal, optical, mechanical and dielectric properties of nonlinear optical crystal 4-methoxy benzaldehyde-N-methyl 4-stilbazolium tosylate," Journal of Physics and Chemistry of Solids 72, pp. 1002-1007, 2011.

[8] H. O. Marcy, M. J. Roskar, L. F. Warren, and P. H. Cunningham, "L-histidine tetrafluoroborate: a solution-grown semiorganic crystal for nonlinear frequency conversion," Optical Letters, vol. 20, no. 3, pp. 252-254, 1995.

[9] S. Natarajan, S. A. M. Britto and E. Ramachandran, "Growth, thermal, spectroscopic and optical studies of L-alanium maleate, a new organic nonlinear optical material," Crystal Growth and Design, vol. 6, pp. $137-140,2006$

[10] J. T. Prakash and S. Kumararaman, "Growth and characterization of L-proline cadmium chloride monohydrate single crystals," Materials Letters, vol. 62, pp. 4097-4099, 2008.

[11] V. S. Dhanya, M. R. Sudarsanakumar, S. Suma, S. Prasanna, K. R. Babu, B. S. Kumar, and S. M. Roy, "Growth and characterization of a new polymorph of lead succinate: A promising NLO material," Journal of Crystal Growth, vol. 319, pp. 96-101, 2011.

[12] V. Chithambaram, S. J. Das, R. A. Nambi, and S. Krishnan, "Synthesis, growth and characterization of novel semiorganic nonlinear optical potassium boro-succinate (KBS) single crystals," Optics \& Laser Technology, vol. 43, pp. 1229-1232, 2011.

[13] P. Anandan and R. Jayavel, "Crystal growth and characterization of semiorganic single crystals of L-histidine family for NLO applications," Journal of Crystal Growth, vol. 322, pp. 69-73, 2011.

[14] K. Prabha, B. Feng, H. Chen, G. Bhagavannarayana, and P. Sagayaraj, "Growth and characterization of a new non-linear optical tris lead tris barium borate (TLTBB) single crystal," Materials Chemistry and Physics, vol. 127, pp. 79-84, 2011.

[15] M. L. Caroline and S. Vasudevan, "Growth and characterization of an organic nonlinear optical material: L-alanine alaninium nitrate," Materials Letters, vol. 62, pp. 2245-2248, 2008.

[16] A. J. A. Pragasam, S. Selvakumar, K. Thamizharasan, D. Prem Anand, and P. Sagayaraj, "Growth and Optical characterization of $\mathrm{Cu}^{-}$and $\mathrm{Mg}$ " substituted L-arginine di phosphate single crystals," Journal of Crystal Growth, vol. 280, pp. 271-278, 2005.

[17] J. Ramajothi and S. Danuskodi, "Crystal growth, thermal and optical studies on a semiorganic nonlinear material for blue-green laser generation," Spectrochimica Acta Part A, vol. 68, pp. 1213-1219, 2007.

[18] S. K. Kurtz and T. T. Perry, "A powder technique for the evaluation of non-linear optical materials," Applied Physics, vol. 39,pp. 3798-3813, 1968.

[19] H. A. Petrosyan, R. A. Apreyan, A. A. Hovhannesyan, A. K. Atanesyan, and A. M. Petrosyan, "Nonlinear Optical Activity and Spectroscopy of L-Nitrohistidine Monohydrate," Journal of Contemporary, vol. 44, 43-49, 2009.

[20] T. J. Prakash, N. Vijayan, and S. Kumararaman, "Growth and characterization studies on $\mathrm{g} 1 \mathrm{y} \mathrm{c}$ i $\mathrm{n}$ e barium dihydrochloride single crystals for NLO applications," Spectrochimica Acta Part A, vol. 71, pp. 1250-1252, 2008. 